\title{
A QUANTITATIVE RISK ASSESSMENT OF SINONASAL CANCER AS A FUNCTION OF TIME IN WORKERS OCCUPATIONALLY EXPOSED TO WOOD DUST
}

\section{RENATA SOĆKO}

Nofer Institute of Occupational Medicine, Łódź, Poland

Department of Chemical Safety

\begin{abstract}
Objectives: The aim of this study is to conduct a quantitative assessment of the risk related to occupational exposure to wood dust and the occurrence of sinonasal epithelial cancer (SNEC) by histotype, depending on the duration of exposure, using mathematical models. Material and Methods: The relationship between the occurrence of SNEC by histotype (adenocarcinoma [AR] and tumors of other histotypes) and exposure to wood dust has been studied using the values of the odds ratios for individual periods of employment involving exposure to wood dust, and their $95 \%$ confidence intervals. The dose-response curves were constructed (more precisely, the duration of exposure-response curves). The author attempted to match the linear, quadratic or exponential models. Results: In all SNEC cases, there is a relationship between the duration of occupational exposure to wood dust and the relative risk of developing cancer. The estimated relative risk of developing AR after 35 years of exposure to wood dust is about 300 , and the estimated risk of developing SNEC is 50, compared to non-exposed people for whom the relative risk is equal to 1 . However, the relative risk of developing other types of cancer is $<20$, also in comparison with non-exposed people. Conclusions: The author has identified a relationship between the duration of occupational exposure to wood dust and the relative risk of developing cancer in all SNEC cases. Int J Occup Med Environ Health. 2021;34(4):541-9
\end{abstract}

Key words:

occupational exposure, risk assessment, wood dust, mathematical models, carcinogenicity, nasal cancer

\section{INTRODUCTION}

Dust generated from wood processing is one of the identified occupational and carcinogenic agents. It is estimated that around 3.6 million working people are exposed to wood dust in EU countries [1]. Based on epidemiological studies, including case-control studies, a relationship has been demonstrated between the incidence of nasal cancer or sinonasal epithelial cancer (SNEC) and exposure to both soft and hard wood dust [2,3]).

Over three-quarters of all sinonasal cancer (SNC) cases are malignant neoplasms, among which the most popular ones are sinonasal adenocarcinoma (AD) and sinonasal squamous cell carcinoma (SCC) [4-8].

\footnotetext{
Funding: this work was supported by the Nofer Institute of Occupational Medicine (project No. IMP 24.20 entitled "Indication of the most common occupational chemical carcinogens and mutagens among women - spatial analysis of their occurence and development of recommendations aiming to reduction of women's exposure underestimation," project manager: Agnieszka Niepsuj, M.Sc.) and by the Ministry of Science and Higher Education/National Center for Research and Development (fifth stage of the multi-annual program financed in 2020-2022 entitled "Improving safety and working conditions," program coordinator: Central Institute for Labor Protection - National Research Institute).

Received: June 4, 2020. Accepted: November 19, 2020.

Corresponding author: Renata Soćko, Nofer Institute of Occupational Medicine, Department of Chemical Safety, św. Teresy 8, 91-348 Łódź, Poland (e-mail: Renata.Socko@imp.lodz.pl).
} 
Conducted studies have indicated that occupational exposure to wood dust may also be associated with an increased risk of other tumors, for example, nasopharyngeal [9] or lung [10] cancer.

The meta-analysis carried out has indicated a relationship between exposure to wood dust and an increased risk of nasal cancer, but when it comes to nasopharyngeal or lung cancer, the results remain inconclusive [4,6,7,9-11].

Some inconsistencies can be explained by the different duration of exposure to wood dust, the varied latency of the development of a particular type of cancer, and the difficulty in designing studies to identify the increased risk of cancer in a given study group. In addition, certain non-professional factors, such as the environment, genetic material, lifestyle and smoking habits, have a disruptive impact on the test results. Therefore, the author performed a quantitative tumor risk assessment for all SNEC, AD, and other histotypes of cancer, excluding nasopharyngeal and lung cancer.

\section{All SNEC types}

\section{Adenocarcinoma}

A meta-analysis by Binazzi et al. [8], taking into account 11 cohort studies and 17 case-control studies, confirmed the relationship between exposure to wood dust and a significant increase in the incidence of SNC. The relative risk (RR) of developing SNC was: $\mathrm{RR}_{\text {pooled }}=5.91,95 \%$ confidence interval (CI): 4.31-8.11 for the case-control studies, and 1.61, 95\% CI: 1.10-2.37 for the cohort studies. The strongest associations concerned $\mathrm{AD}\left(\mathrm{RR}_{\text {pooled }}=\right.$ 29.43, 95\% CI: 16.46-52.61). A significant risk was also identified for SCC $\left(\mathrm{RR}_{\text {pooled }}=1.46,95 \%\right.$ CI: 1.01-2.1 $)$ [8]. A higher risk of developing SNC was observed among people exposed to wood dust, compared to the general population (the odds ratio $[\mathrm{OR}]=2.83,95 \% \mathrm{CI}$ : 1.88-4.27). Two histotypes of SNC, AD and SCC, were identified. The risk of developing $\mathrm{AD}$ was high $(\mathrm{OR}=$ 7.78, 95\% CI: 2.97-20.25), whereas the risk of developing SNC and SCC was lower.
The relationship between the occurrence of SNEC by histotype ( $\mathrm{AD}$ and other histotypes) and the duration of exposure to wood dust was also studied by d'Errico et al. [12]. The authors presented the OR and $95 \% \mathrm{CI}$ values for the groups exposed to wood dust for 5, 10, 15, 20, 25, 30 and 35 years. The OR values of the occurrence of all examined cancer types for particular periods of employment involving exposure to wood dust, and also their $95 \%$ CIs, increased with the duration of exposure. These studies showed the highest risk of developing $\mathrm{AD}$.

The relationship between $\mathrm{AD}$ and exposure to wood dust, especially hard wood dust, is more pronounced than in SCC. The risk of developing AD is $10-50 \%$ higher in comparison to other types of cancer of the nasal cavity and paranasal sinuses (referred to as SNC).

The research results obtained from 12 case-control studies (described by Demers et al. [13]), indicate a significant risk of developing $\mathrm{AD}$ in men employed in furniture factories $(\mathrm{OR}=13.5,95 \% \mathrm{CI}: 9.0-20.0)$; with the risk being particularly significant for carpenters (OR $=41.1,95 \% \mathrm{CI}$ : 24.5-68.7). A moderate risk of developing $\mathrm{AD}$ was reported in sawmill workers (OR $=19.7,95 \%$ CI: 11.1-35.1). For men, the relationship between the increased risk of developing $\mathrm{AD}$ and exposure to wood dust (OR $=0.6,95 \%$ CI: $0.1-4.7$ for low exposure, $\mathrm{OR}=3.1,95 \% \mathrm{CI}$ : $1.6-6.1$ for medium exposure, and $\mathrm{OR}=45.5,95 \% \mathrm{CI}: 28.3-72.9$ for high exposure) was observed. The following 4 exposure categories were created: zero exposure, and low, medium or high exposure, corresponding approximately to the respective estimated concentration values, i.e., concentration equal to 0 , concentration of $<1 \mathrm{mg} / \mathrm{m}^{3}$, concentration of $1-5 \mathrm{mg} / \mathrm{m}^{3}$, and concentration of $>5 \mathrm{mg} / \mathrm{m}^{3}$ [13].

In the study by Demers et al. [14], a significant relationship was demonstrated between the risk of developing $\mathrm{AD}$ and exposure. The risk analysis as a function of the duration of exposure showed a significant increase in the $\mathrm{OR}$ of $\mathrm{AD}$ with a longer duration of exposure among men. The OR for the first year of exposure 
was $1.08,95 \% \mathrm{CI}: 1.07-1.09$, for $<5$ years of work: $\mathrm{OR}=5.3,95 \% \mathrm{CI}: 2.5-11.1$, and for 10-19 years of work: $\mathrm{OR}=10.7,95 \%$ CI: $5.2-11.8$. For the duration of exposure of $\geq 30$ years, the risk was very high and the OR value reached 36.7, 95\% CI: 22.0-61.3 [14].

The above data indicate a long latency of the development of $\mathrm{AD}$ in men, i.e., approximately 20 years. For women employed under similar conditions involving exposure to wood dust, the results were inconclusive. There was a smaller increase in the risk of developing $\mathrm{AD}(\mathrm{OR}=$ 2.78, 95\% CI: $0.75-10.3)$. As in men, the risk of developing $\mathrm{AD}$ was higher in women employed in furniture factories $(\mathrm{OR}=4.6,95 \% \mathrm{CI}: 1.16-18.3)$ [14]. The analysis of these studies took into account the workplace and period of employment. Workplaces were classified into the following 3 categories by probability of exposure to wood dust: possible exposure, probable exposure and definite exposure. A significant increase in the number of deaths due to nasal cancer was recorded (11 cases; the standardized mortality ratio $[\mathrm{SMR}]=3.1,95 \% \mathrm{CI}$ : 1.6-5.6).

The RR of death due to SNCby category of exposure towood dust, for all wood workers, was as follows: possible exposure: 1 case of death; $\mathrm{SMR}=0.8 ; 95 \%$ CI: $0.0-4.6$, probable exposure: 1 case of death, SMR = 1.2, 95\% CI: 0.0-6.5, and definite exposure: 9 cases; SMR = 8.4, 95\% CI: 3.9-16.0. An increased risk of developing SNEC was demonstrated among people employed in furniture production while no deaths due to this type of cancer were reported in employees of plywood plants. An increased risk of cancer was also reported in people who had started working before 1940 ( 9 cases; SMR $=12.5,95 \%$ CI: 5.7-23.7) and in those employed under exposure conditions for $>30$ years $(8$ cases, SMR $=7.6,95 \%$ CI: 3.3-15.0). Moreover, 3 cases of cancer were reported in people employed under exposure conditions for $>20$ years (i.e., 20-29 years) $(\mathrm{SMR}=2.6,95 \% \mathrm{CI}$ : 0.5-7.6) [14].

Alonso-Sardon et al. [7] also showed a relationship between $\mathrm{AD}$ and exposure to wood dust, based on a meta-analysis that included clinical case-control and cohort studies. Overall, 22 studies on nasal AD were included in the analysis. A statistically significant increase in the risk of developing nasal $\mathrm{AD}$ was demonstrated in workers exposed to wood dust (OR $=10.28,95 \%$ CI: 5.92-17.85, $\mathrm{p}<0.0001)$.

A significantly increased risk of developing $\mathrm{SNC}$ was observed after exposure to wood dust. The OR for SNC was 7.78 (95\% CI: 2.97-20.25) as shown in AD studies [6]. Gordon et al. [15] described the meta-analysis results regarding the risk of nasal cancer in various professions related to woodworking, which are based on the results of 12 case-control tests considered reliable. The average OR of SNC for male wood workers was equal to 2.6 (95\% CI: 2.1-3.3), and the highest risk of developing such cancer occurred in furniture industry workers, for whom the RR of nasal $\mathrm{AD}$ amounted to 29.

\section{Squamous cell carcinoma}

Compared to $\mathrm{AD}$, the results for SCC were more ambiguous. The risk of developing this type of cancer, especially in women, was about twice as high under exposure to medium and high levels of wood dust. In women, a relationship was also found between the magnitude of effects and the duration of exposure. These studies, however, were conducted on a small population of women. For men, the risk of developing SCC was not related to the type of work they performed, or to the duration or amount of exposure, and it was generally lower in comparison to the risk of developing $\mathrm{AD}$.

The study results not included in the analysis by Demers et al. [16] showed that exposure to softwood dust posed a greater risk of sinonasal SCC than exposure to hardwood dust.

Siew et al. [4] studied the risk of nasal cancer among Finnish male populations exposed to wood dust from various tree species. The risk of nasal cancer in those subjects was: $\mathrm{RR}=1.59,95 \%$ CI: $1.06-2.38$, with nasal SCC being most frequently noted $(\mathrm{RR}=1.98,95 \% \mathrm{CI}: 1.19-3.31)$. 
In turn, Zhang et al. [6] did not report a significant increase in the risk of SCC in people exposed to wood dust (OR $=1.18,95 \%$ CI: 0.64-2.19).

The presented research results seem to prove the relationship between exposure to hardwood dust and $\mathrm{AD}$, or between exposure to softwood dust and SCC of the nasal mucosa and paranasal sinuses, although the risk appears higher under exposure to mixed dust. Based on this evidence, the International Agency for Research on Cancer includes all wood dust (hard and soft) in group 1, i.e., the substance which is carcinogenic to humans [3].

The level of exposure to wood dust has not been assessed in the available literature. Instead, the ratios of the chances of developing cancer among exposed people are only presented in relation to non-exposed ones. Therefore, there is a problem with the quantitative assessment of cancer risk as a result of exposure to wood dust (mainly hard dust). For this reason, the risk is assessed on a binary basis in all existing publications, i.e., only the fact of exposure itself, or its absence, is taken into account.

In the work by Demers et al. [14], the SMRs were presented for a cohort of furniture and plywood industry workers in England and the USA, with the English and Welsh population used as reference. The SMR value (e.g., for furniture industry workers) of paranasal sinus cancer was 2.9 (95\% CI: 1.2-5.9). As regards the quantitative risk assessment, no conclusions could be drawn as no quantitative exposure assessment was presented. What is more, the authors were even not sure if the data only concerned exposure to wood dust.

In the work by Demers et al. [13], the results of some case-control studies were described. The ORs for those cancer cases that were caused by practicing occupations involving exposure to wood dust were presented, but no exposure assessment was performed. However, an attempt was made to estimate the concentration levels. A similar situation concerned the work by Demers et al. [15] and the meta-analysis prepared by Zhang et al. [6].

An attempt to assess the actual risk is found in the work by d'Errico et al. [12], in which the authors presented the ORs for the groups exposed to wood dust for 5 years (Table 1). The amount of exposure was not determined, and the only criterion was the period of employment involving exposure. By using the ORs in individual periods of employment, and their $95 \%$ CIs, the so-called doseresponse curves were constructed. However, these merely reflected the RR values which were treated as a multiple of the risk of developing cancer in comparison to the nonexposed population.

A similar approach was taken by Hildesheim et al. [17], but the results were presented only for 2 periods of exposure, i.e., $\leq 10$ years and $>10$ years; therefore, d'Errico et al. [12] used these results in their estimations.

\section{MATERIAL AND METHODS}

To estimate the risk of developing nasal cancer in workers exposed to wood dust, the occupational history data of these people was used, collected by hospitals in Italy and described in the work by d'Errico et al. [12].

The relationship between the risk of developing SNEC by histotype (AD and cancer of other histotypes) and exposure to wood dust was studied using the OR values for individual periods of occupational exposure to wood dust, and their $95 \%$ CIs. The SPSS ver. 22 software was used to perform the calculations.

Providing the OR values for the 5-year exposure groups (Table 1) means that, for example, it is not known how to treat a person exposed for 2.5 or 7.5 years. To avoid such a problem, the author of this article tried to build a continuous function based on d'Errico's results.

Dose-response curves were built (more precisely, the duration of exposure-response curves). The author of this work attempted to match the linear, quadratic and exponential models for each type of cancer (Table 2). 
Table 1. The odds ratios (ORs) and their 95\% confidence intervals (CIs) depending on the duration of exposure to wood dust for cancer types associated with this exposure in workers exposed to wood dust, based on data collected by hospitals in Italy and described in the work by d'Errico et al. [12]

\begin{tabular}{lccc}
\hline \multirow{2}{*}{ Exposure duration } & \multicolumn{3}{c}{ OR $(95 \% \mathrm{CI})$} \\
\cline { 2 - 4 } & SNEC & $\mathrm{AD}$ & other histotypes \\
\hline No exposure & 1.00 & 1.00 & 1.00 \\
5 years & $1.77(1.46-2.14)$ & $2.24(1.76-2.85)$ & $1.51(1.22-1.87)$ \\
10 years & $3.10(2.13-4.56)$ & $5.00(3.09-8.10)$ & $2.30(1.49-3.48)$ \\
15 years & $5.50(3.11-9.74)$ & $11.2(5.42-23.04)$ & $3.40(1.82-6.48)$ \\
20 years & $9.70(4.53-20.80)$ & $25.0(9.53-65.55)$ & $5.20(2.22-12.09)$ \\
25 years & $17.1(6.61-44.42)$ & $55.9(16.74-186.5)$ & $7.80(2.70-22.54)$ \\
30 years & $30.2(9.64-94.86)$ & $124.9(29.4-530.7)$ & $11.8(3.30-42.03)$ \\
35 years & $53.4(14.07-202.6)$ & $279.3(51.67->999)$ & $17.8(4.02-78.37)$ \\
\hline
\end{tabular}

AD - adenocarcinoma; SNEC - sinonasal epithelial cancer.

Table 2. Determination coefficients for fitted OR regression models for cancers associated with exposure to wood dust

\begin{tabular}{|c|c|c|c|c|c|c|c|c|c|}
\hline \multirow{3}{*}{ Model } & \multicolumn{9}{|c|}{$\mathrm{R}^{2}$} \\
\hline & \multicolumn{3}{|c|}{ SNEC } & \multicolumn{3}{|c|}{$\mathrm{AD}$} & \multicolumn{3}{|c|}{ other histotypes } \\
\hline & $\begin{array}{l}\text { regression } \\
\text { line }\end{array}$ & $\begin{array}{l}\text { lower } \\
\text { limit }\end{array}$ & $\begin{array}{l}\text { upper } \\
\text { limit }\end{array}$ & $\begin{array}{l}\text { regression } \\
\text { line }\end{array}$ & $\begin{array}{l}\text { lower } \\
\text { limit }\end{array}$ & $\begin{array}{l}\text { upper } \\
\text { limit }\end{array}$ & $\begin{array}{l}\text { regression } \\
\text { line }\end{array}$ & $\begin{array}{l}\text { lower } \\
\text { limit }\end{array}$ & $\begin{array}{l}\text { upper } \\
\text { limit }\end{array}$ \\
\hline Linear & 0.825 & 0.909 & 0.743 & 0.725 & 0.827 & 0.741 & 0.894 & 0.972 & 0.801 \\
\hline Quadratic & 0.986 & 0.997 & 0.968 & 0.963 & 0.987 & 0.980 & 0.995 & 1.000 & 0.982 \\
\hline Exponential & 1.000 & 1.000 & 1.000 & 1.000 & 1.000 & 0.997 & 1.000 & 1.000 & 1.000 \\
\hline
\end{tabular}

Abbreviations as in Table 1.

The lower and upper limits indicate the $\mathrm{R}^{2}$ values for the exponential regression models for the lower and upper end of the $95 \%$ CIs of the ORs presented in Table 1.

Table 3. Regression coefficients of the exponential models describing the relationship between the duration of exposure to wood dust and the relative risk of developing cancer

\begin{tabular}{lcccccc}
\hline \multirow{2}{*}{ Variable } & \multicolumn{2}{c}{ SNEC } & \multicolumn{2}{c}{ AD } & \multicolumn{2}{c}{ Other histotypes } \\
\cline { 2 - 7 } & $\beta_{0}$ & $\beta_{1}$ & $\beta_{0}$ & $\beta_{1}$ & $\beta_{0}$ & $\beta_{1}$ \\
\hline Regression line & 0.999 & 0.114 & 1.002 & 0.161 & 1.002 & 0.082 \\
Lower confidence limit & 0.998 & 0.076 & 1.001 & 0.113 & 1.001 & 0.040 \\
Upper confidence limit & 1.001 & 0.152 & 1.126 & 0.200 & 1.002 & 0.125 \\
\hline
\end{tabular}

Abbreviations as in Table 1.

The lower and upper confidence limits are the exponential regression models for the lower and upper end of 95\% CIs of the OR values presented in Table 1. 

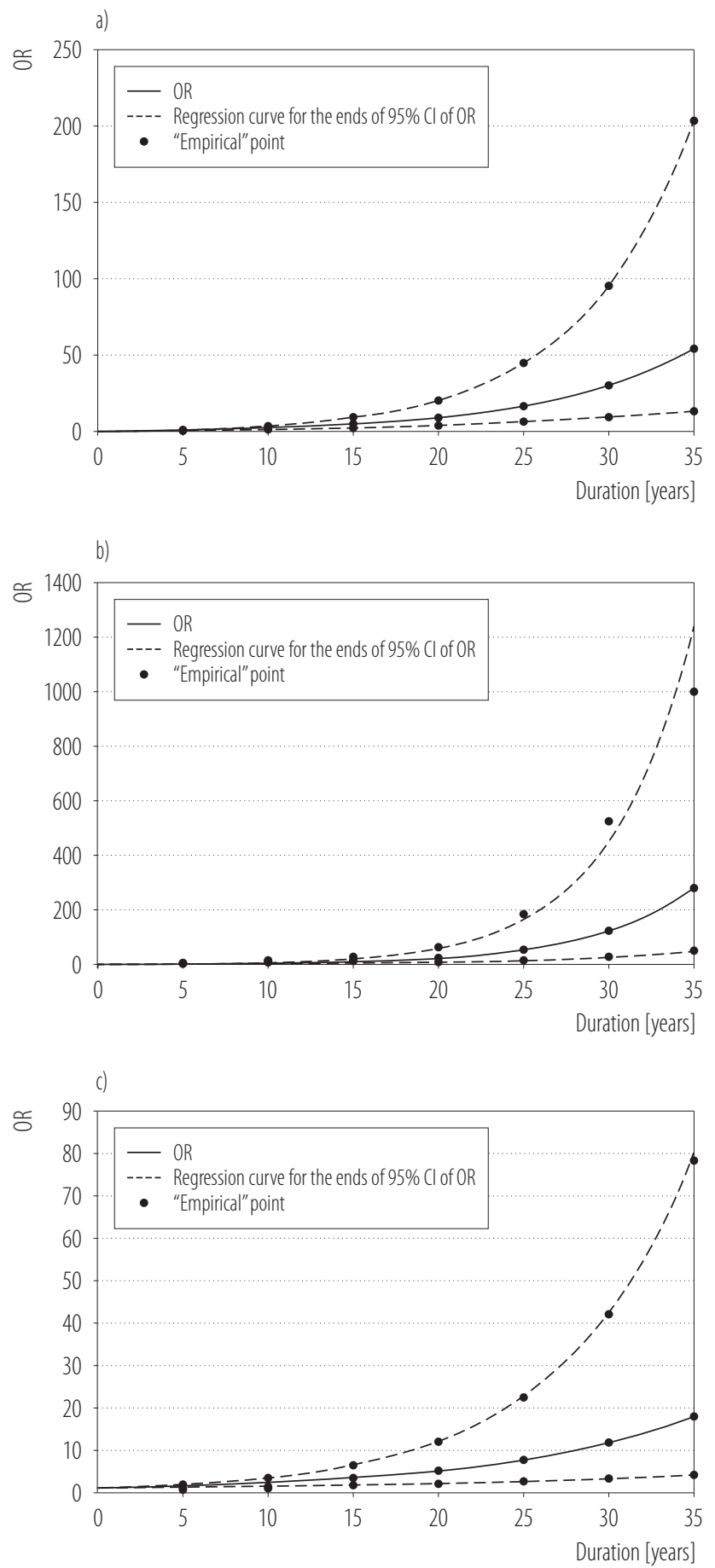

The empirical points come from d'Errico et al. [12].

Figure 1. The relationship between the odds ratio (OR) of occurrence of: a) sinonasal epithelial cancer, b) adenocarcinoma, and c) other cancer histotypes, and the duration of exposure to wood dust in workers
The linear model (due to the simplicity of risk calculation):

$$
\mathrm{OR}=\beta_{0}+\beta_{1} \times \mathrm{t}
$$

The quadratic model:

$$
\mathrm{OR}=\beta_{0}+\beta_{1} \times \mathrm{t}+\beta_{2} \times \mathrm{t}^{2}
$$

The exponential model:

$$
\mathrm{OR}=\beta_{0} \times \exp \left(\beta_{1} \times \mathrm{t}\right)
$$

where:

$\mathrm{t}$ - the duration of exposure in years. As the criterion for assessing the quality of the model's fit to empirical data, the author adopted the value of the $\mathrm{R}^{2}$ coefficient of determination,

$\beta_{0}$ - the regression coefficient, the intercept in the regression equation,

$\beta_{1}, \beta_{2}$ - the regression coefficients for the duration of exposure $\left(\beta_{1}\right)$ and the square value of the duration of exposure $\left(\beta_{2}\right)$.

\section{RESULTS}

\section{Modeling results}

The exponential model shows the best fit to empirical data, so it provided the basis for building the dose-response relationship. The values of the coefficients are presented in Table 3. The relationship between the ORs of developing SNEC, AD and other cancer histotypes, and the duration of exposure to wood dust, are presented in the graphic images of the curves (Figure 1a, b and c).

\section{Example of risk calculation}

Assuming that a person worked for 7 years and 9 months, i.e., 7.75 years, if this value is used in the formula, together with coefficients $\beta_{0}$ and $\beta_{1}$ (Table 3), the RR values as shown in Table 4 are obtained:

- for SNEC: $\mathrm{OR}=0.999 \times \exp (0.114 \times 7.75)=2.42$;

- for $\mathrm{AD}: \mathrm{OR}=1.002 \times \exp (0.1617 .75)=3.49$;

- for other histotypes: $\mathrm{OR}=1.002 \times \exp (0.0827 .75)=1.89$. 
Table 4. The relative risk expressed as the odds ratios (ORs) of developing a specific type of cancer after exposure to wood dust lasting 7 years and 9 months ( 7.75 years), estimated using the exponential model

\begin{tabular}{lccc}
\hline \multirow{2}{*}{\multicolumn{1}{c}{ Variable }} & & RR & \\
\cline { 2 - 4 } & SNEC & AD & other histotypes \\
\hline OR & 2.42 & 3.49 & 1.89 \\
Lower confidence limit OR* & 1.80 & 2.40 & 1.36 \\
Upper confidence limit OR* & 3.25 & 5.31 & 2.64 \\
\hline
\end{tabular}

Abbreviations as in Table 1.

* The lower and upper confidence limits are the exponential regression models for the lower and upper end of the $95 \%$ CIs of the OR values presented in Table 1.

The estimated values of the RR of developing a given type of cancer, after exposure to wood dust for 7 years and 9 months, range between the respective estimated values for 5-year and 10-year periods of exposure.

In the case of $\mathrm{SNEC}, \mathrm{AD}$ and other histotypes of cancer, there is a relationship between the duration of occupational exposure to wood dust and the RR of developing cancer. The estimated RR of AD after 35 years of exposure to wood dust is about 300, and that of SNEC about 50 , compared to non-exposed people for whom the RR is equal to 1 . However, the RR of other types of cancer is $<20$ in comparison to non-exposed people.

\section{DISCUSSION}

An increased risk of nasal cancer due to occupational exposure to wood dust has been sufficiently described in the literature. However, as the author of this work has already mentioned, there is no quantitative assessment of exposure to this dust, and the only criterion is the duration of occupational exposure. The authors of previous studies have only determined whether the effect of exposure to wood dust is manifested as developing cancer or not $[13,14]$. A risk assessment was conducted by d'Errico et al. [12]. These authors presented the ORs in groups with 5-year occupational exposure to wood dust, showing that the duration of exposure to this dust is an important factor in the development of nasal cancer, as it determines the magnitude of cancer risk. The longer the duration of exposure to wood dust, the more the OR for nasal cancer increases.

The author of this work, using the values of the ORs and their $95 \%$ CIs (given in the work by d'Errico et al. [12]) for individual periods of employment, attempted to match the linear, quadratic and exponential models in order to estimate the risk not only in the 5-year exposure groups but also for any other periods of exposure to wood dust, with exposure lasting 7 years and 9 months being used as the example. The exponential model turned out to be best suited to empirical data and became the basis for estimating the risk of nasal cancer for any duration of exposure to wood dust.

\section{CONCLUSIONS}

The risk of SNEC and 2 other histotypes of cancer was significantly related to prior exposure to wood dust. The dose-response relationship, or more precisely the duration of exposure-response relationship, was observed in people exposed to wood dust. The estimated RR of developing SNEC after 35 years of exposure to wood dust was found to reach 50, compared to non-exposed persons for whom the RR was equal to 1 . The RR of developing other histotypes was $<20$. The highest risk was reported for $\mathrm{AD}$, reaching about 300 . The OR values set for exposure lasting of 7 years and 9 months are between the ORs set for 5-year and 10-year periods of exposure. This implies that, 
together with the increasing duration of exposure, even in the short term, the risk of exposure to wood dust also increases.

\section{ACKNOWLEDGMENTS}

The author of this work would like to thank Wiesław Szymczak, Ph.D., Prof. of the University of Lodz, for his comprehensive assistance in statistical analysis.

\section{REFERENCES}

1. Kauppinen T, Vincent R, Liukkonen T, Grzebyk M, Kauppinen A, Welling I, et al. Occupational exposure to inhalable wood dust in the members states of the European Union. Ann Occup Hyg. 2006;50(6):549-61, https://doi.org/10.1093/ annhyg/mel013.

2. International Agency for Research on Cancer (IARC). Monographs on the evaluation of carcinogenic risks to humans. Wood dust and formaldehyde. Lyon: IARC; 1995.

3. International Agency for Research on Cancer (IARC). Monographs on the evaluation of the carcinogenic risk of chemicals to humans. Arsenic, metals, fibres and dusts. Lyon: IARC; 2012.

4. Siew SS, Kauppinen T, Kyyrönen P, Heikkilä P, Pukkala E. Occupational exposure to wood dust and formaldehyde and risk of nasal, nasopharyngeal, and lung cancer among Finnish men. Cancer Manag Res. 2012;4:223-32, https://doi.org/ 10.2147/CMAR.S30684.

5. Siew SS, Martinsen JI, Kjaerheim K, Sparén P, Tryggvadottir L, Weiderpass E, et al. Occupational exposure to wood dust and risk of nasal and nasopharyngeal cancer: A case-control study among men in four nordic countries - With an emphasis on nasal adenocarcinoma. Int J Canc. 2017;141:2430-6, https://doi.org/10.1002/ijc.31015.

6. Zhang JX, Xu H, Shen T, Zhu QX. Wood dust exposure and risk of sinonasal and nasopharyngeal cancer: a meta-analysis. Aus J Derm. 2014;1(2):1-5.

7. Alonso-Sardón M, Chamorro AJ, Hernández-García I, Iglesiasde-Sena H, Martín-Rodero H, Herrera C, et al. Association be- tween occupational exposure to wood dust and cancer: a systematic review and meta-analysis. PLOS ONE. 2015;10(7):e0133024, https://doi.org/10.1371/journal.pone.0133024.

8. Binazzi A, Ferrante P, Marinaccio A. Occupational exposure and sinonasal cancer: a systematic review and meta-analysis. BMC Cancer. 2015;15:49-65, https://doi.org/10.1186/s12885015-1042-2.

9. Beigzadeh Z, Pourhassan B, Kalantary S, Golbabaei F. Occupational exposure to wood dust and risk of nasopharyngeal cancer: Asystematic review and meta-analysis. Environ Res. 2018;171:170-6, https://doi.org/10.1016/j.envres. 2018.12.022.

10. Vallières E, Pintos J, Parent ME, Siemiatyc J. Occupational exposure to wood dust and risk of lung cancer in two population-based case-control studies in Montreal, Canada. Environ Health. 2015;14:1-9, https://doi.org/10.1186/1476-069X-14-1. 11. Vaughan TL, Stewart PA, Teschke K, Lynch CF, Swanson GM, Lyon JL, et al. Occupational exposure to formaldehyde and wood dust and nasopharyngeal carcinoma. Occup Environ Med. 2000;57:376-84, https://doi.org/10.1136/oem.57.6.376.

12. d'Errico A, Pasian S, Baratti A, Zanelli R, Alfonzo S, Gilardi L, et al. A case-control study on occupational risk factors for sino-nasal cancer. Occup Environ Med. 2009;66:448-55, https://doi.org/10.1136/oem.2008.041277.

13. Demers PA, Kogevinas M, Boffetta P, Leclerc A, Luce D, Gerin M, et al. Wood dust and sino-nasal cancer: pooled reanalysis of twelve case-control studies. Am J Ind Med. 1995;28(2):151-66, https://doi.org/10.1002/ajim.4700280202.

14. Demers PA, Boffetta P, Kogevinas M, Blair A, Miller BA, Robinson CF, et al. Pooled reanalysis of cancer mortality among five cohorts of workers in woodrelated industries. Scand J Work Environ Health. 1995;21:179-90, https://doi. org/10.5271/sjweh.26.

15. Demers PA, Teschke K, Kennedy SM. What to do about softwood? A review of respiratory effects and recommendations regarding exposure limits. Am J Ind Med. 1997;31(4): 385-98, https://doi.org/10.1002/(sici)1097-0274(199704)31:4 $<385$ ::aid-ajim3>3.0.co;2-v. 
16. Gordon I, Boffetta P, Demers PA. A case study comparing a meta-analysis and a pooled analysis of studies of sinonasal cancer among wood workers. Epidemiology. 1998;9(5): $518-24$.
17. Hildesheim A, Dosemeci M, Chan CC, Chen CJ, Cheng YJ, Hsu MM, et al. Occupational exposure to wood, formaldehyde, and solvents and risk of nasopharyngeal carcinoma. Cancer Epidemiol Biomarkers Prev. 2001;10:1145-53.

This work is available in Open Access model and licensed under a Creative Commons Attribution-NonCommercial 3.0 Poland License - http://creativecommons.org/ licenses/by-nc/3.0/pl/deed.en. 